\title{
Cognitive-Enhancing Effect of Quercetin in a Rat Model of Parkinson's Disease Induced by 6-Hydroxydopamine
}

\author{
Napatr Sriraksa, ${ }^{1}$ Jintanaporn Wattanathorn, ${ }^{2}$ Supaporn Muchimapura, ${ }^{2}$ \\ Somsak Tiamkao, ${ }^{3}$ Kamoltip Brown, ${ }^{4}$ and Kowit Chaisiwamongkol ${ }^{4}$ \\ ${ }^{1}$ Department of Physiology and Graduate School (Neuroscience Program), Faculty of Medicine, Khon Kaen University, \\ Khon Kaen 40002, Thailand \\ ${ }^{2}$ Department of Physiology, Faculty of Medicine, Khon Kaen University, Khon Kaen 40002, Thailand \\ ${ }^{3}$ Department of Medicine, Faculty of Medicine, Khon Kaen University, Khon Kaen 40002, Thailand \\ ${ }^{4}$ Department of Anatomy, Faculty of Medicine, Khon Kaen University, Khon Kaen 40002, Thailand
}

Correspondence should be addressed to Jintanaporn Wattanathorn, jinwat05@gmail.com

Received 3 March 2011; Revised 1 May 2011; Accepted 25 May 2011

Academic Editor: José Luis Ríos

Copyright (C) 2012 Napatr Sriraksa et al. This is an open access article distributed under the Creative Commons Attribution License, which permits unrestricted use, distribution, and reproduction in any medium, provided the original work is properly cited.

\begin{abstract}
Oxidative stress has been reported to induce cognitive impairment in Parkinson's disease. This paper aimed to determine the effect of quercetin, a substance possessing antioxidant activity, on the cognitive function in a rat model of Parkinson's disease. Male Wistar rats, weighing 200-250 g, were orally given quercetin at doses of $100,200,300 \mathrm{mg} / \mathrm{kg}$ BW once daily for a period of 14 days before and 14 days after the unilateral lesion of right substantia nigra induced by 6-hydroxydopamine (6-OHDA). Their spatial memory was assessed at 7 and 14 days of treatment and neuron density was determined, malondialdehyde (MDA) level, the activity of superoxide dismutase (SOD), catalase (CAT), and glutathione peroxidase (GPx) were evaluated at the end of the experiment. In addition, the activity of acetylcholinesterase (AChE) was also measured. It was found that all doses of quercetin enhanced spatial memory. Therefore, it is suggested that the cognitive-enhancing effect of quercetin occurs partly because of decreased oxidative damage resulting in increased neuron density.
\end{abstract}

\section{Introduction}

Parkinson's disease (PD) is the second most common neurodegenerative disease. To date, the precise mechanism responsible for pathogenesis of $\mathrm{PD}$ is not well understood. Accumulating lines of evidence have demonstrated that oxidative stress plays a crucial role on the pathogenesis of PD [1-5]. The motor impairment observed in patients seems to be the most notable point of concern when prescribing medicine or planning treatment. In recent years, it was indicated that nonmotor deficits became an important part of patient management [6]. It has been known that patients in the early stages of PD already have impairment of cognitive performance and this deficit in turn gives rise to a poor quality of life $[7,8]$ and economic burden [9]. Recently, it was found that 6-hydroxydopamine (6-OHDA) was recognized as a good model for early stages of PD, especially in terms of emotional and cognitive deficits [10]. Although the substantia nigra is the brain area that has been indicated to be the most vulnerable to neurodegeneration in PD and mediates the clinical manifestation (rigidity, resting tremor, slowness of voluntary movement, and postural instability), but other brain regions such as locus ceruleous, raphe neuclei, nucleus basalis of Meynert, and the brain area that responsible for cognition, hippocampus, were also affected. Previous MRI studies clearly revealed that decreased hippocampal volume accompanied the cognitive impairment in $\mathrm{PD}$ patients [11-13]. Based on the role of oxidative stress on the pathophysiology of $\mathrm{PD}$, the neuroprotective and cognitive enhancing effects of substances possessing antioxidant activity have gained much attention.

Quercetin (QC) is a polyphenolic compound found in common vegetables and fruits such as onions, broccoli, and apples. Previous studies have demonstrated that quercetin 


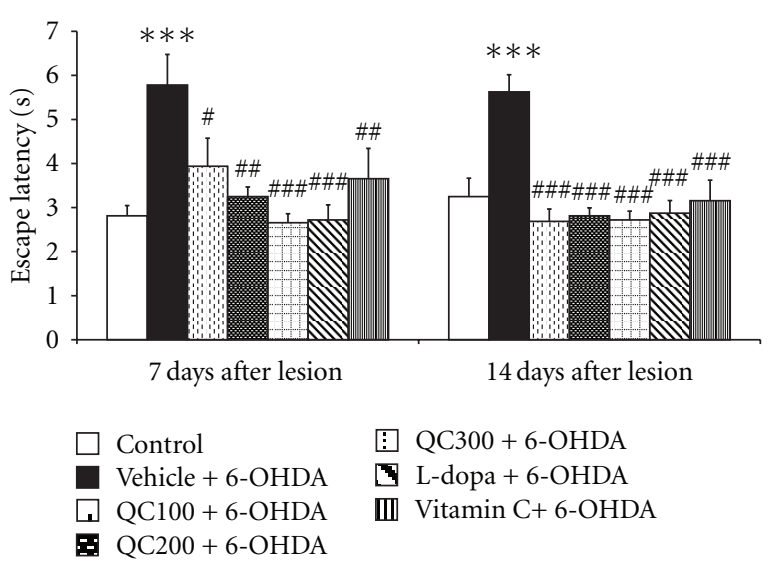

FIGURE 1: Effect of quercetin on escape latency in the Morris water maze test in an animal model of Parkinson's disease induced by 6OHDA. Data were expressed as mean \pm S.E.M. for 8 rats in each group. ${ }^{* *} P<0.001$ compared to control; ${ }^{*} P<0.05$; ${ }^{\# \#} P<0.01$; ${ }^{\# \# \#} P<0.001$ compared to the vehicle +6 -OHDA-treated group.

contains many good biological properties for human health including antioxidant [14], anti-inflammation [15], and anticancer [16] activities. Recently, it has been reported that quercetin can pass through the blood-brain barrier of in situ models [17]. In addition, quercetin exerts the protective effect in a stroke model induced by transient global ischemia [18]. Quercetin significantly protected the neuronal cells from the oxidative stress-induced neurodegeneration in Alzheimer's disease [19], decreased lipid peroxidation, improved the activity of catalase and superoxide dismutase [20] and also prevented glutathione depletion [21]. Previous study showed that the EGb761, a standardized extract from the herbal medicine Ginkgo biloba, contains a high amount of quercetin and exhibits the neuroprotective effect against oxidative damage induced by 6-OHDA [22]. Moreover, it was found that quercetin attenuated the neuronal death in the hippocampus resulting in improved learning and memory in arm maze test [23]. Therefore, these pieces of evidence point out the possibility that quercetin might exert an influence on the central nervous system.

Based on the antioxidant and anti-inflammatory actions of QC, we hypothesized that QC might mitigate the neurotoxicity and cognitive impairment in an animal model of PD induced by 6-OHDA. To elucidate this issue, the current study aimed to determine the effects of QC on spatial memory and neuron density in the hippocampus of an animal model of PD induced by 6-OHDA. In addition, the possible underlying mechanism was also investigated.

\section{Materials and Methods}

2.1. Animals. Adult male Wistar rats (10 weeks old) were obtained from the National Laboratory Animal Center, Salaya, Nakhon Pathom. The animals weighted between 200 to 250 grams at the beginning of experiment. They were housed 5 per cage and maintained in 12:12 light: dark cycle

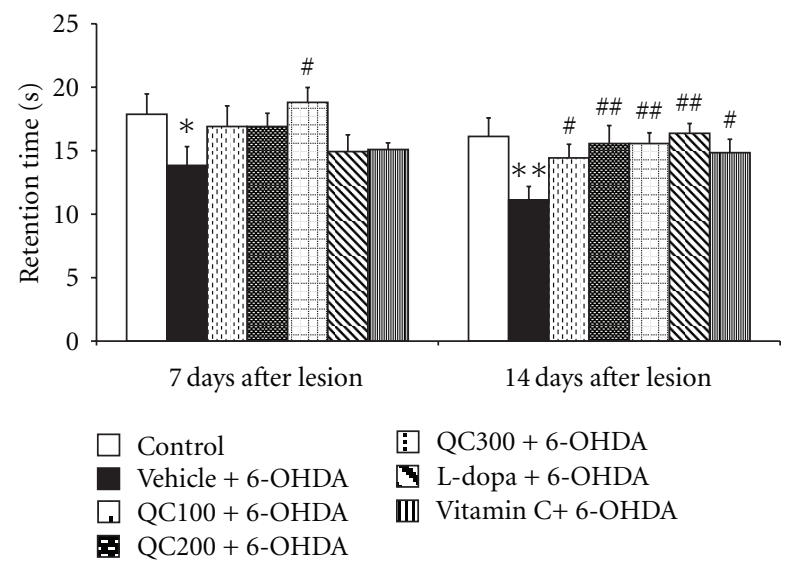

Figure 2: Effect of quercetin on retention time in the Morris water maze test in an animal model of Parkinson's disease induced by $6-$ OHDA. Data were expressed as mean \pm S.E.M. for 8 rats in each group. ${ }^{*} P<0.05$; ${ }^{* *} P<0.01$ compared to control; ${ }^{\#} P<0.05$; ${ }^{\#} P<$ 0.01 compared to the vehicle +6 -OHDA-treated group.

and given access to food and water ad libitum. The experiments were performed to minimize animal suffering in accordance with the internationally accepted principles for laboratory use and care of European Community (EEC directive of 1986; 86/609/EEC). The experimental protocols were approved by the Institutional Animal Care and Use Committee.

2.2. Drugs and Chemicals. Quercetin was purchased from Sigma-Aldridge Company. All chemical substances used in this study were analytical grade. Both Levodopa (L-dopa), a standard drug used for PD treatment, and vitamin C, an antioxidant possessing the neuroprotective and cognitive enhancing effects, were used as positive controls in this study. Normal saline solution (NSS) was used as vehicle throughout the study. All administered substances were freshly prepared.

2.3. Experimental Protocol. The animals were randomly divided into 7 groups ( $n=8$ animals/group) as described below.

Group I: Control. Animals were not treated with any drugs or injections.

Group II: Vehicle plus 6-OHDA injection. Animals were treated with NSS once daily via oral route for 14 days before and 14 days after the injection of 6-OHDA into the right substantia nigra.

Groups III-V: Rats were treated with quercetin at various doses ranging from 100,200, and $300 \mathrm{mg} / \mathrm{kg}$ BW once daily via oral route for 14 days before and 14 days after the injection of 6-OHDA into the right substantia nigra.

Groups VI-VII: Rats were orally treated with either Levodopa (L-dopa; $5 \mathrm{mg} / \mathrm{kg} \mathrm{BW}$ ) or vitamin C $(200 \mathrm{mg} / \mathrm{kg}$ ) once daily for 14 days before and 14 days after the lesion of the right substantia nigra induced by 6-OHDA.

Each rat in all groups, except the control group, was orally given the same volume of substance suspension for 14 days. The rats were then subjected to 6-OHDA injection 


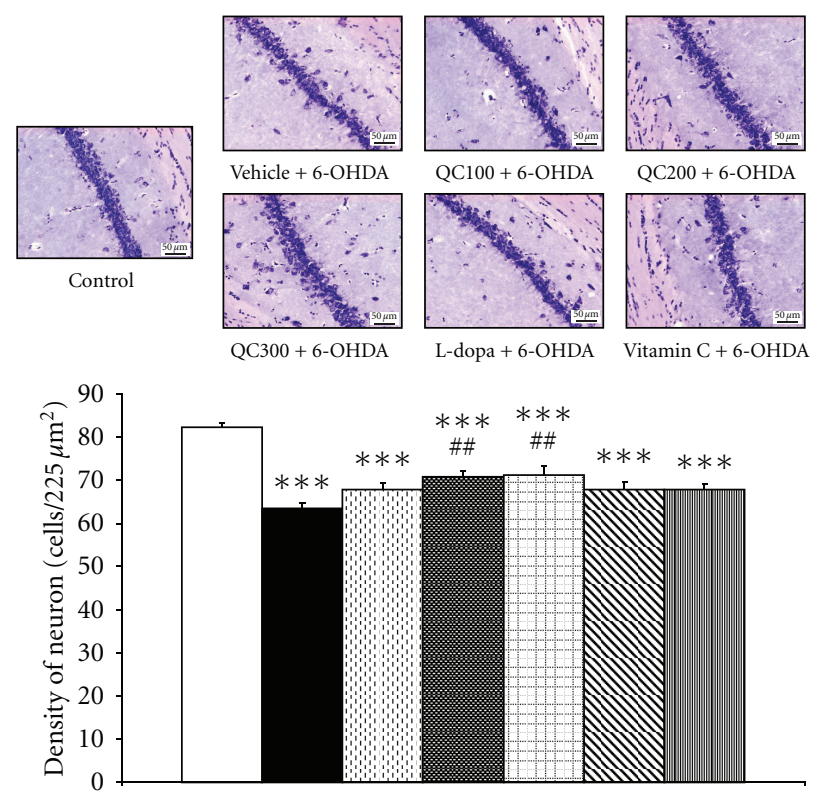

(a)

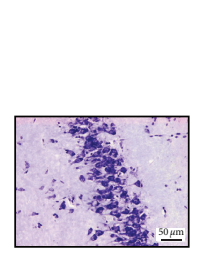

Control
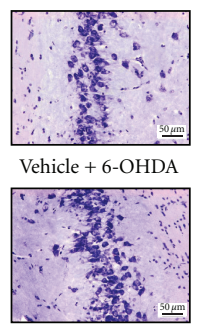

QC300 + 6-OHDA

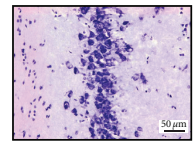

$\mathrm{QC100+6-OHDA}$

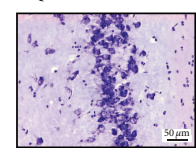

L-dopa + 6-OHDA Vitamin C + 6-OHDA

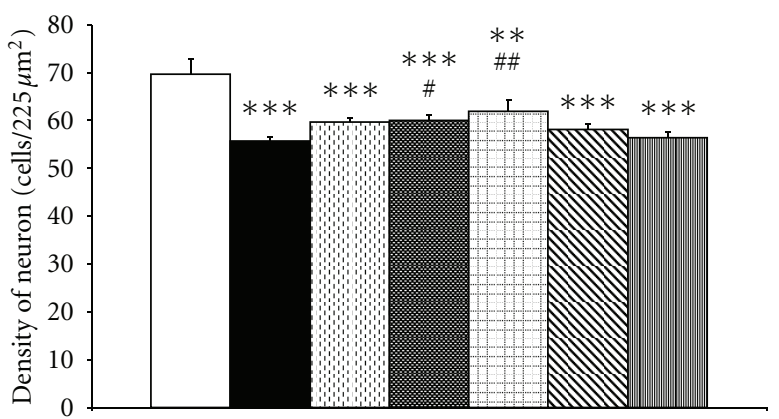

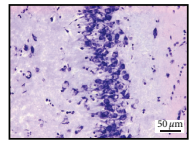

$\mathrm{QC} 200+6-\mathrm{OHDA}$

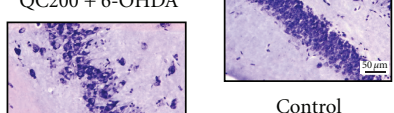

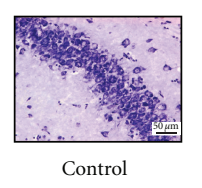
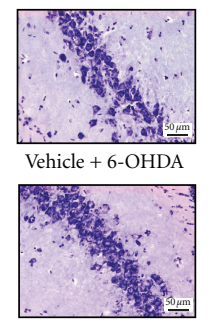

$\mathrm{QC} 300+6-\mathrm{OHDA}$

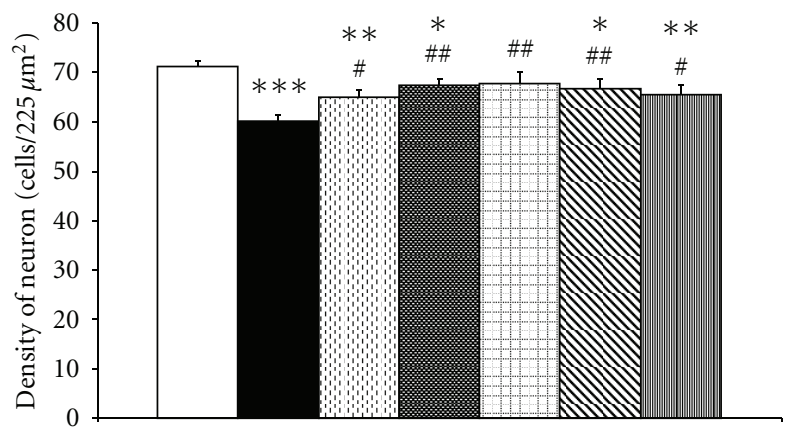

(b)

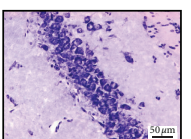

$\mathrm{QC} 100+6-\mathrm{OHDA}$
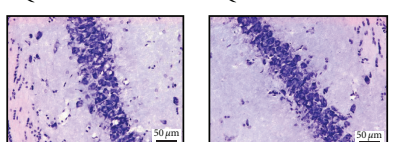

Vitamin C + 6-OHDA
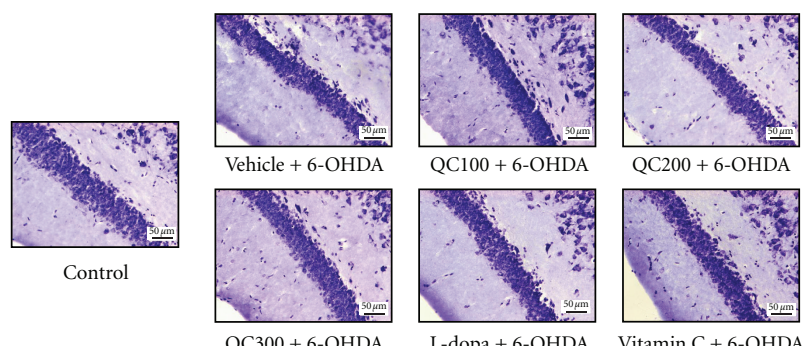

QC200 + 6-OHDA
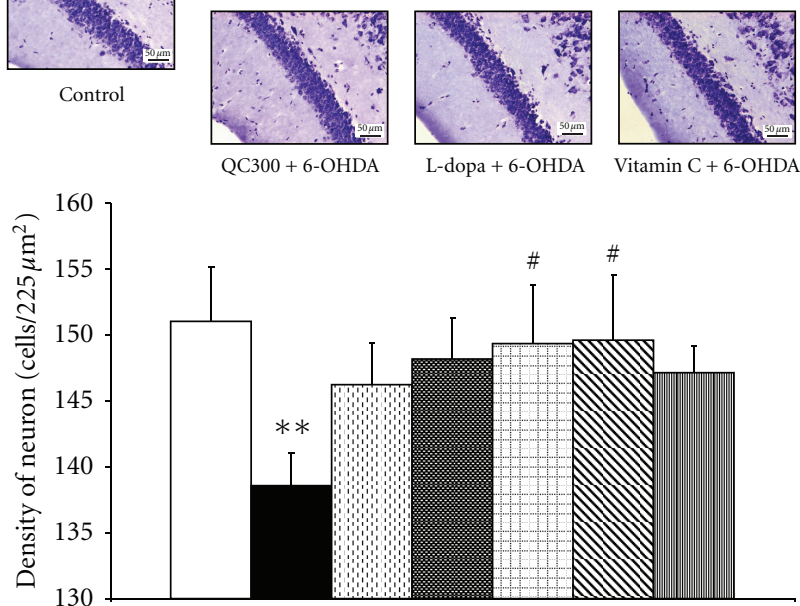

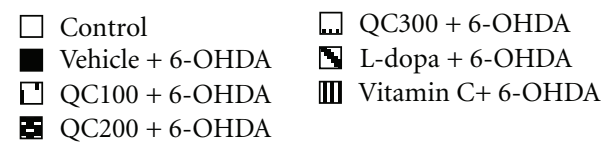

(c)

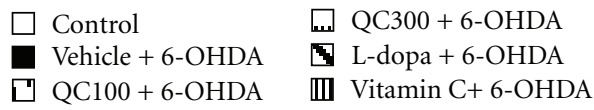

용 QC200+6-OHDA

(d)

FIGURE 3: Effect of quercetin on neuron density in various subregions of the hippocampus $(\mathrm{a}=\mathrm{CA} 1 ; \mathrm{b}=\mathrm{CA} 2 ; \mathrm{c}=\mathrm{CA}$; $\mathrm{d}=\mathrm{Dentate}$ gyrus $)$ in an animal model of Parkinson's disease induced by 6-OHDA. Scale bars $50 \mu \mathrm{m}$, the magnification was $40 \mathrm{X}$. Data were expressed as mean \pm S.E.M. for 8 rats in each group. ${ }^{*} P<0.05 ;{ }^{* *} P<0.01 ;{ }^{* * *} P<0.001$ compared to control; ${ }^{\#} P<0.05 ;{ }^{\#} P<0.01$ compared to the vehicle + 6-OHDA-treated group. 

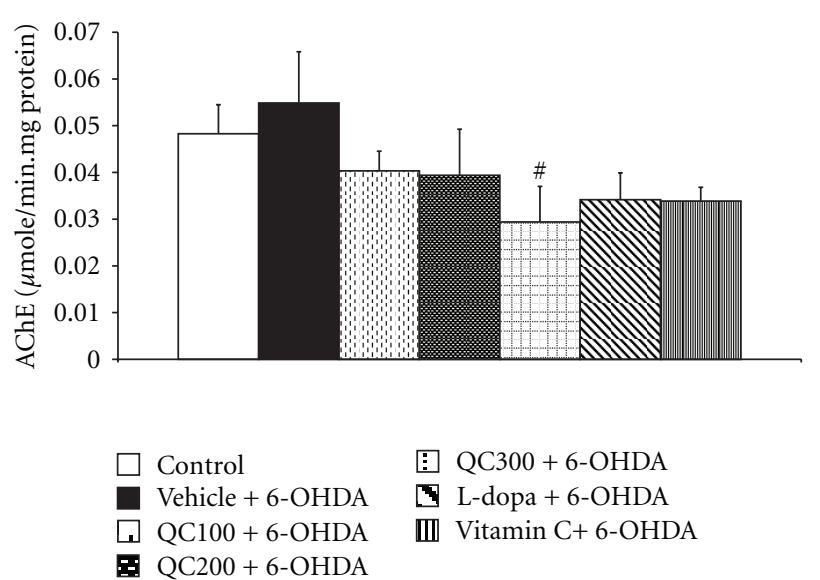

FIGURE 4: Effect of quercetin on the activity of acetylcholinesterase (AChE) in the hippocampus in an animal model of Parkinson's disease induced by 6-OHDA. Data were expressed as mean \pm S.E.M. for 8 rats in each group. ${ }^{\#} P<0.05$ compared to the vehicle +6 OHDA-treated group.
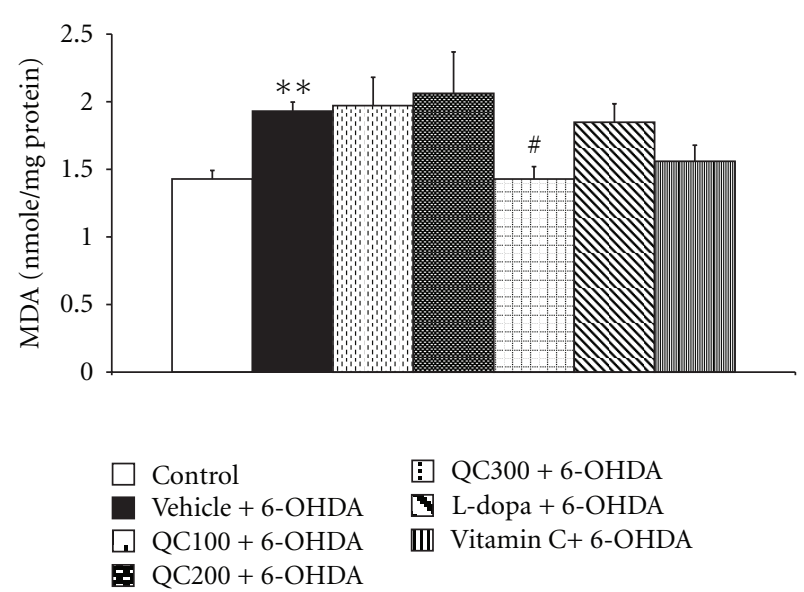

Figure 5: Effect of quercetin on the level of malondialdehyde (MDA) in the hippocampus in an animal model of Parkinson's disease induced by 6-OHDA. Data were expressed as mean \pm S.E.M. for 8 rats in each group. ${ }^{* *} P<0.01$ compared to control; ${ }^{*} P<0.05$ compared to the vehicle +6 -OHDA-treated group.

using the stereotaxic apparatus. The 6-OHDA infusion was performed using a Hamilton syringe and rats were allowed to recover after the lesion for 6 days. They were then tested for spatial memory using Morris water maze test at 7 and 14 days after 6-OHDA injection. At the end of experiment, rats were sacrificed and their brains were removed and the density of survival neurons, the activity of acetylcholinesterase (AChE), and scavenger enzymes including superoxide dismutase (SOD), catalase (CAT), and glutathione peroxidase (GPx) in the hippocampus were determined. Moreover, the malondialdehyde (MDA) level in the mentioned area was also determined.

2.4. The Administration of 6-OHDA. 6-OHDA was prepared as described previously by Ferro and coworkers [24]. Rats

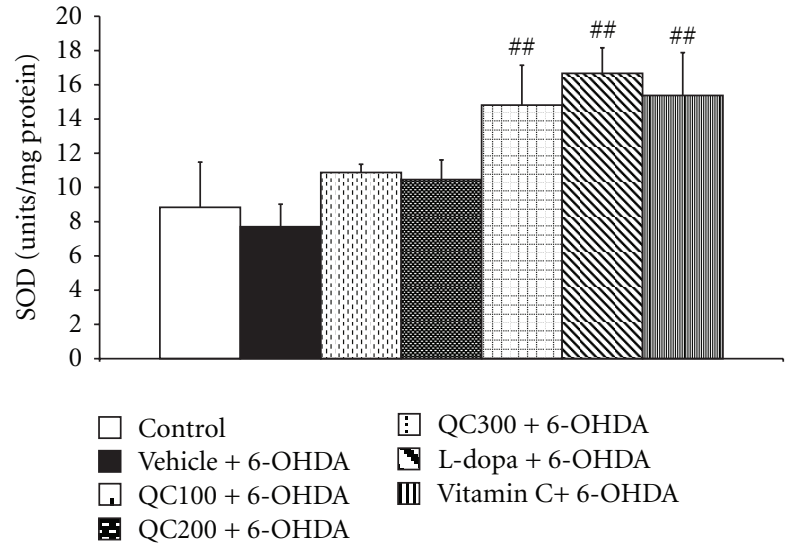

FIGURE 6: Effect of quercetin on the activity of superoxide dismutase (SOD) in the hippocampus in an animal model of Parkinson's disease induced by 6-OHDA. Data were expressed as mean \pm S.E.M. for 8 rats in each group. ${ }^{\# \#} P<0.01$ compared to the vehicle +6 OHDA-treated group.

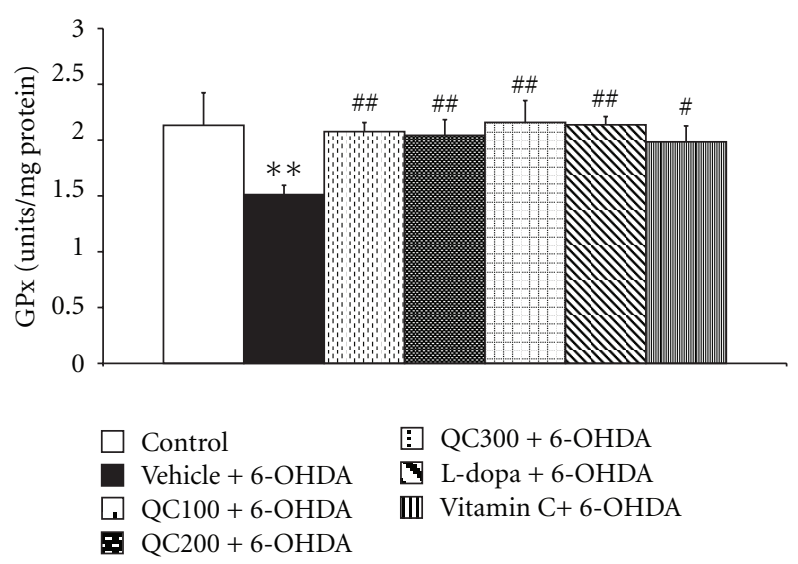

FIgURE 7: Effect of quercetin on the activity of glutathione peroxidase (GPx) in the hippocampus in an animal model of Parkinson's disease induced by 6-OHDA. Data were expressed as mean \pm S.E.M. for 8 rats in each group. ${ }^{* *} P<0.01$ compared to control; ${ }^{\#} P<0.05$; ${ }^{\#} P<0.01$; compared to the vehicle + 6-OHDAtreated group.

were anesthetized with $50 \mathrm{mg} / \mathrm{kg}$ sodium thiopental (i.p.), each animal was mounted on a stereotaxic stand, the skin overlying the skull was cut to expose the skull, and the coordinates for the substantia nigra par compacta (SNpc) were accurately measured (anteroposterior $-0.5 \mathrm{~mm}$ from bregma, mediolateral $2.1 \mathrm{~mm}$ from midline, and dorsoventral $-7.7 \mathrm{~mm}$ from the skull). The $6 \mu \mathrm{g}$ of $6-\mathrm{OHDA}$, which dissolved in $2 \mu \mathrm{L} 0.2 \%$ ascorbic acid saline, were perfused into $\mathrm{SNpc}$ through a 30-gauge stainless needle. After the surgery, animals were allowed to recover from anesthesia and then placed in their cages.

2.5. Morris Water Maze Test. The water maze consisted of a metal pool $(170 \mathrm{~cm}$ in diameter $\times 58 \mathrm{~cm}$ tall $)$ filled with tap water $\left(25^{\circ} \mathrm{C}, 40 \mathrm{~cm}\right.$ deep $)$. The pool was divided into 

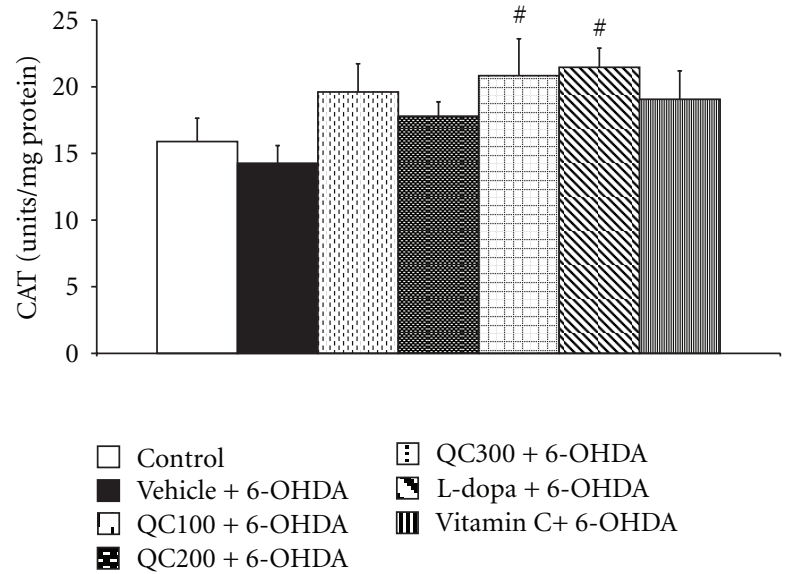

FIGURE 8: Effect of quercetin on the activity of catalase (CAT) in the hippocampus in an animal model of Parkinson's disease induced by 6-OHDA. Data were expressed as mean \pm S.E.M. for 8 rats in each group. ${ }^{\#} P<0.05$ compared to the vehicle +6 -OHDA-treated group.

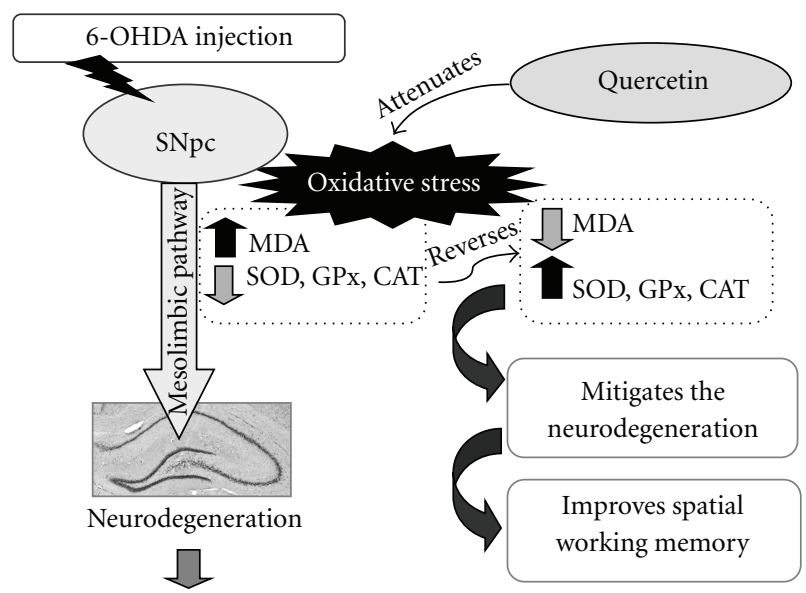

Cognitive impairment

Figure 9: Hypothetical diagram of the possible underlying mechanism for the cognitive enhancing effect of quercetin on an animal model of Parkinson's disease induced by 6-OHDA.

4 quadrants (NE, NW, SE, and SW) by two imaginary lines crossing the center of the pool. The water surface was covered with nontoxic milk. The removable platform was placed below the water level at the center of one quadrant. For each animal, the location of the invisible platform was placed at the center of one quadrant and remained there throughout training. The rats must memorize the platform location in relation to various environmental cues because the location of the escape platform in and outside the pool is not visible. Each rat was gently placed in the water facing the wall of the pool from one of the four starting points $(\mathrm{N}, \mathrm{E}, \mathrm{S}$, or $\mathrm{W})$ along the perimeter of the pool, and the animal was allowed to swim until it found and climbed onto the platform. During training session, the rat was gently placed on the platform when it could not reach the platform in 60 seconds. In either case, the subject was left on the platform for 15 seconds and then removed from the pool. The time for animals to climb on the hidden platform was recorded as escape latency or acquisition time. In order to determine the capability of the animals to retrieve and retain information, the platform was removed $24 \mathrm{hr}$ later and the rat was released into the quadrant diagonally opposite to that which contained the platform. Time spent in the region that previously contained the platform was recorded as retention time. In each trial, the animal was quickly dried with towel before being returned to the cage [25].

2.6. Histological Procedure. Following anesthesia with sodium pentobarbital $(50 \mathrm{mg} / \mathrm{kg} \mathrm{BW})$, the brain fixation was carried out by transcardial perfusion with a fixative solution containing $4 \%$ paraformaldehyde in $0.1 \mathrm{M}$ phosphate buffer $\mathrm{pH}$ 7.3. After the perfusion, the brains were removed and stored overnight in a fixative solution that is used for perfusion. They were then infiltrated with $30 \%$ sucrose solution at, and kept at, $4{ }^{\circ} \mathrm{C}$. The specimens were frozen rapidly and the coronal sections at $30 \mu \mathrm{M}$ thick were prepared using cryostat. All sections were rinsed in the phosphate buffer and picked up on slides coated with $0.01 \%$ aqueous solution of a high molecular weight poly L-lysine.

2.7. Nissl Staining. The duplicate coronal sections of brains were stained with $0.75 \%$ cresyl violet, dehydrated through graded alcohols $(70,95,100 \% 2 \mathrm{x})$, placed in xylene, and mounted using DPX.

2.8. Determination of Acetylcholinesterase, Superoxide Dismutase, Glutathione Peroxidase and Catalase Activity, and the Malondialdehyde Level. The rats were divided into various groups as previously described in the experimental protocol. After the last dose of administration, all rats were sacrificed. The hippocampus of the lesion side was isolated and prepared as a homogenate to determine the MDA level and the activities of AChE, SOD, CAT, and GPx. MDA was estimated by determining the accumulation of thiobarbituric acid reactive substances (TBARS) [26] in the hippocampal homogenate whereas the activities of acetylcholinesterase [27], superoxide dismutase [28], glutathione peroxides [29], and catalase [30] were determined using the colorimetric method.

2.9. Statistical Analysis. Data are expressed as means \pm S.E.M. and were analyzed statistically by one-way ANOVA, followed by post hoc (LSD) test. The results were considered statistically significant at $P$ value $<0.05$.

\section{Results}

3.1. Morris Water Maze Test. Cognitive impairment is commonly observed in Parkinson patients. Therefore, we examined the effect of quercetin on spatial memory by using the Morris water maze test and recorded escape latency and retention time as the indices. The results were shown in Figures 1 and 2. Figure 1 demonstrates that 6-OHDA 
injection significantly increased escape latency $(P<0.001$ compared to control). Both L-dopa and vitamin $C$ could decrease escape latency throughout the observation period (day 7: $P<0.001$ and 0.01 , respectively, compared to vehicle+6-OHDA; day 14: $P<0.001$ all, compared to vehicle+6-OHDA). At the 7th day after lesion, all doses of quercetin $(100,200$, and $300 \mathrm{mg} / \mathrm{kg} \mathrm{BW})$ used in this study significantly decreased the escape latency $(P<0.05,0.01$, and 0.001 , resp., compared to vehicle+6-OHDA). The beneficial effect of quercetin to improve learning and memory was also observed throughout the 14-day period after lesion $(P$ $<0.001$ all, compared to vehicle+6-OHDA).

Figure 2 shows that 6-OHDA injection significantly decreased retention time $(P<0.05$ and 0.01 at day 7 and day 14 , resp., compared to control). However, only the high dose of quercetin significantly increased retention time at 7 days after lesion $(P<0.05$ compared to vehicle+6-OHDA). When the treatment was prolonged further to 14 days after lesion, all doses of quercetin-treated groups could enhance retention time $(P<0.05,0.01,0.01$ resp., compared to vehicle+6OHDA) as similar as the positive modulation effect of Ldopa and vitamin $C(P<0.01$ and 0.05 resp., compared to vehicle+6-OHDA).

3.2. Density of Survival Neuron in the Hippocampus. Previous information reported that the hippocampus plays a crucial role in learning and memory. Therefore, we also determined the effect of quercetin on the neuron density in the various subregions (CA1, CA2, CA3, and dentate gyrus) of the hippocampus. The results are shown in Figure 3. 6-OHDA injection significantly produced the neurodegeneration in all subregions of the hippocampus $(P<0.001,0.001,0.001$, and 0.01 for CA1, CA2, CA3, and the dentate gyrus resp., compared to control). L-dopa-treated group significantly increased the neuron density in both CA2 and the dentate gyrus $(P<0.01$ and 0.05 resp., compared to vehicle+6OHDA) while vitamin C-treated group showed a significant increase of neuron density only in CA2 $(P<0.05$ compared to vehicle+6-OHDA). Quercetin at a dose of $300 \mathrm{mg} / \mathrm{kg} \mathrm{BW}$ significantly increased neuron density in all subregions of the hippocampus $(P<0.01,0.01,0.01$, and 0.05 resp., compared to vehicle+6-OHDA). The medium dose of quercetin $(200 \mathrm{mg} / \mathrm{kg} \mathrm{BW})$ significantly enhanced neuron density in CA1, CA2, and CA3 $(P<0.01,0.01$ and 0.05 resp., compared to vehicle+6-OHDA), while the low dose or quercetin at dose of $100 \mathrm{mg} / \mathrm{kg} \mathrm{BW}$ could significant increase the neuron density in only CA2 $(P<0.05$ compared to vehicle+6OHDA).

3.3. Acetylcholinesterase Activity. Based on the knowledge that cholinergic system plays the crucial role in spatial memory, the effect of quercetin on the activity of AChE, an indirect indicator of cholinergic function was evaluated. It was found that the high dose of quercetin significantly decreased the AChE activity in the hippocampal homogenate $(P<0.05$ compared to vehicle+6-OHDA; Figure 4$)$. However, the low and medium doses $(100$ and $200 \mathrm{mg} / \mathrm{kg} \mathrm{BW}$, resp.) of quercetin and both positive control groups (Ldopa and vitamin $\mathrm{C}$ treatments) failed to produce significant alterations on this enzyme activity.

3.4. Determination of Malondialdehyde Level and Scavenging Enzymes Activities. The right hippocampus was isolated in order to determine MDA levels and scavenging enzyme activities. The results are shown in Figures 5, 6, 7, and 8 . It was found that 6-OHDA injection significantly increased MDA level $(P<0.01$ compared to control) but decreased the activity of GPx $(P<0.01$ compared to control). Both positive-control-treated groups failed to show a significant reduction of MDA levels in the hippocampus while the high dose of quercetin used in this study significantly decreased MDA levels in the mentioned area $(P<0.05$ compared to vehicle+6-OHDA). In addition, both L-dopa and vitamin $C$ also increased the activities of SOD $(P<0.01$ all, compared to vehicle+6-OHDA), GPx $(P<0.01$ and 0.05 resp., compared to vehicle+6-OHDA). However, only rats subjected to Ldopa showed significantly increased CAT activity $(P<0.05$ compared to vehicle+6-OHDA). Interestingly, it was found that the rats subjected to the high dose of quercetin had significantly enhanced SOD, CAT, and GPx activity in the hippocampus $(P<0.01,0.01$, and 0.05 resp., compared to vehicle+6-OHDA). The medium and low doses of quercetintreated groups also had significantly increased GPx activity in the aforementioned area $(P<0.05$ all, compared to vehicle+6-OHDA).

\section{Discussion}

The results of this study clearly show the cognitive enhancing effect of quercetin in this PD model. It was found that treatment with quercetin for two weeks before and after a 6-OHDA injection can improve performance in the Morris water maze test of the rats.

6-OHDA is one of the most common neurotoxins used in experiments in order to mimic Parkinsonism in rodents. The possible underlying mechanism of neurotoxicity induced by 6-OHDA has been reported to be related to the oxidative stress caused by the production of hydroxyl radicals during autoxidation [31-33] and the inhibition of complex I [34] resulting in excessive oxidative stress and leading to neuronal death. Previous studies demonstrated that 6-OHDA could produce the cognitive deficit in animals [35-39], and oxidative stress has been shown to play an important role in memory impairment [40]. Thus, 6-OHDA is suitable to be used to mimic Parkinsonian and also cognitive impairment in rats (see Figure 9).

It is well known that the substantia nigra is the area of the brain that is most affected in PD but other brain areas are also affected. The hippocampus is the brain area that plays an important role in spatial memory [41, 42], thus we determined the cognitive performance of the rats using the Morris water maze test which is the best tool to determine spatial learning and memory in rodents [43].

We found that quercetin significantly improved the cognitive impairment induced by 6 -OHDA injection, indicated 
by the decrease of time spent to find out platform (escape latency) and the increase of retention time.

It has been reported that there was a tight correlation between cognitive impairment in PD and cholinergic deficit [44-46]. Therefore, we also determined the activity of AChE, an indirect indicator to evaluate the cholinergic system. The results of our study demonstrated that quercetin in a high dose significantly decreased AChE activity in the hippocampal homogenate, which indicates that there is the increase of available acetylcholine at the synaptic terminal resulting in the improvement of cognitive performance by the animals.

It was found that in Parkinson patients' brain, there is an increase of lipid peroxidation $[47,48]$ and a decrease in the level of free radical scavenging enzymes [49]. The oxidative stress-induced neuronal death is the manner of 6-OHDA model. Therefore, we evaluated the density of survival neurons in the hippocampus. The results demonstrated that quercetin attenuated the neurodegeneration in all subregions of the hippocampus. In addition, we also determined the level of MDA and the activities of scavenging enzymes. The results of the present study demonstrate that 6-OHDA injection causes a significant increase of MDA levels but causes a significant decrease of scavenging enzymes indicating that free radicals are effectively involved in the development of cognitive impairment in PD.

It is not clear how 6-OHDA produces oxidative stress in the hippocampus. Based on the knowledge that there are four main pathways of dopaminergic system, one of them is mesolimbic pathway which connects between the ventral tegmental area and the limbic system and includes the hippocampus. Moreover, it has been shown that the dentate gyrus of the hippocampus received the dopaminergic projection form the ventral tegmental area (A10) and the substantia nigra (A9) [50, 51].

In the present study, we injected 6-OHDA into the substantia nigra and because the nearby structure ventral tegmental area contains dopaminergic neuron thus these neurons might uptake the toxin into their cells too. Therefore, the dopaminergic connection to the hippocampus might be affected leading to the oxidative stress and cognitive impairment observed in the animals. Even though we observed the neuroprotective effect of quercetin in this study, we could not ignore recent information showing the proneurotoxic effect of quercetin if there is coexposure with methylmercury [52].

However, the results obtained from this study failed to show a dose-dependent manner. The possible explanation could be related to the rapid metabolism of quercetin [53]. Therefore, we observed the positive results only in the highdose-treated group but not in the lower two doses because the active metabolites of quercetin might not be able to reach to therapeutic level and produce the significant effect on the parameters measured by this experiment.

Previous studies strongly emphasized that quercetin is considered as a surpass free-radical scavenging antioxidant [54-56] owing to a high number of hydroxyl groups and an ability to donate electrons or hydrogens, and scavenge hydroxyl groups, hydrogen peroxide, and superoxide anions
[57]. It was found that quercetin reverses the cognitive deficit induced by chronic reserpine administration [58]. The mechanisms behind this action could be a direct or indirect effect of quercetin to scavenge free radicals and oxidize metabolites or from iron or copper chelating and inhibit lipid peroxidation properties $[59,60]$. Quercetin was reported to promote glutathione peroxidase [61], reverse the decrease of antioxidant defense of glutathione peroxidase, catalase, and superoxide dismutase induced by UVA light [62], and inhibit the hydrogen peroxide-induced oxidative damage $[63,64]$.

Our results suggest that the cognitive-enhancing effect of quercetin might be due to its antioxidant effect by promoting the activities of scavenging enzymes to protect the neurons from oxidative damage contributing to the survival of neurons in the hippocampus. However, we also observed a slight decrease in antioxidant enzymes in normal control rats. This is possibly due to the prooxidant effect of quercetin in normal cells [65].

Notably, only quercetin in high doses significantly decreased AChE activity resulting in the increase of available acetylcholine, an important neurotransmitter, which plays the crucial role in learning and memory process. However, the results in this study have also shown the beneficial effect on memory in the lower two doses of quercetin (100 and $200 \mathrm{mg} / \mathrm{kg} \mathrm{BW}$ ) but they failed to produce the significant suppression on AChE. We suggest that the improvement of cognition is likely to be related with an increase of neuron density.

\section{Conclusion}

It was concluded that quercetin exerts the cognitive enhancing effect in this PD model via its antioxidant effect resulting in the promotion of neuron survival. Consequently, the use of quercetin as an adjuvant therapeutic agent for the treatment of cognitive impairment in PD should be considered. However, further investigations are still required.

\section{Acknowledgments}

This paper was supported by the Integrative Complimentary Alternative Medicine Research Group, Khon Kaen University, and The National Research Council of Thailand.

\section{References}

[1] B. Halliwell, "Reactive oxygen species and the central nervous system," Journal of Neurochemistry, vol. 59, no. 5, pp. 16091623, 1992.

[2] P. Jenner, D. T. Dexter, J. Sian, A. H. V. Schapira, and C. D. Marsden, "Oxidative stress as a cause of nigral cell death in Parkinson's disease and incidental Lewy body disease," Annals of Neurology, vol. 32, supplement, pp. S82-S87, 1992.

[3] T. Yoshikawa, "Free radicals and their scavengers in Parkinson's disease," European Neurology, vol. 33, no. 1, pp. 60-68, 1993.

[4] C. W. Olanow and W. G. Tatton, "Etiology and pathogenesis of Parkinson's disease," Annual Review of Neuroscience, vol. 22, pp. 123-144, 1999. 
[5] P. Jenner, "Oxidative stress in Parkinson's disease," Annals of Neurology, vol. 53, supplement 3, pp. S26-S38, 2003.

[6] K. R. Chaudhuri, D. G. Healy, and A. H. V. Schapira, "Nonmotor symptoms of Parkinson's disease: diagnosis and management," Lancet Neurology, vol. 5, no. 3, pp. 235-245, 2006.

[7] A. Schrag, M. Jahanshahi, and N. Quinn, "What contributes to quality of life in patients with Parkinson's disease?" Journal of Neurology Neurosurgery and Psychiatry, vol. 69, no. 3, pp. 308-312, 2000.

[8] P. Martínez-Martín, "Impact of the cognitive impairment on the quality of life in patients with Parkinson's disease," Revista de Neurologia, vol. 43, no. 3, pp. 168-172, 2006.

[9] L. Findley, M. Aujla, P. G. Bain et al., "Direct economic impact of Parkinson's disease: a research survey in the United Kingdom," Movement Disorders, vol. 18, no. 10, pp. 1139$1145,2003$.

[10] M. T. Tadaiesky, P. A. Dombrowski, C. P. Figueiredo, E. Cargnin-Ferreira, C. Da Cunha, and R. N. Takahashi, "Emotional, cognitive and neurochemical alterations in a premotor stage model of Parkinson's disease," Neuroscience, vol. 156, no. 4, pp. 830-840, 2008.

[11] M. P. Laakso, K. Partanen, P. Riekkinen et al., "Hippocampal volumes in Alzheimer's disease, Parkinson's disease with and without dementia, and in vascular dementia: an MRI study," Neurology, vol. 46, no. 3, pp. 678-681, 1996.

[12] P. Riekkinen Jr., K. Kejonen, M. P. Laakso, H. Soininen, K. Partanen, and M. Riekkinen, "Hippocampal atrophy is related to impaired memory, but not frontal functions in nondemented Parkinson's disease patients," NeuroReport, vol. 9, no. 7, pp. 1507-1511, 1998.

[13] R. Camicioli, M. M. Moore, A. Kinney, E. Corbridge, K. Glassberg, and J. A. Kaye, "Parkinson's disease is associated with hippocampal atrophy," Movement Disorders, vol. 18, no. 7, pp. 784-790, 2003.

[14] U. Takahama, "Scavenging of active oxygen by flavonoids," Tanpakushitsu Kakusan Koso, vol. 33, no. 16, pp. 2994-2999, 1988.

[15] A. P. Rogerio, A. Kanashiro, C. Fontanari et al., "Antiinflammatory activity of quercetin and isoquercitrin in experimental murine allergic asthma," Inflammation Research, vol. 56, no. 10, pp. 402-408, 2007.

[16] D. W. Lamson and M. S. Brignall, "Antioxidants and cancer III: quercetin," Alternative Medicine Review, vol. 5, no. 3, pp. 196-208, 2000.

[17] K. A. Youdim, M. Z. Qaiser, D. J. Begley, C. A. Rice-Evans, and N. J. Abbott, "Flavonoid permeability across an in situ model of the blood-brain barrier," Free Radical Biology and Medicine, vol. 36, no. 5, pp. 592-604, 2004.

[18] J. Y. Cho, I. S. Kim, Y. H. Jang, A. R. Kim, and S. R. Lee, "Protective effect of quercetin, a natural flavonoid against neuronal damage after transient global cerebral ischemia," Neuroscience Letters, vol. 404, no. 3, pp. 330-335, 2006.

[19] H. J. Heo and C. Y. Lee, "Protective effects of quercetin and vitamin $\mathrm{C}$ against oxidative stress-induced neurodegeneration," Journal of Agricultural and Food Chemistry, vol. 52, no. 25, pp. 7514-7517, 2004.

[20] T. Mahesh and V. P. Menon, "Quercetin allievates oxidative stress in streptozotocin-induced diabetic rats," Phytotherapy Research, vol. 18, no. 2, pp. 123-127, 2004.

[21] M. Fiorani, R. De Sanctis, P. Menghinello, L. Cucchiarini, B. Cellini, and M. Dacha, "Quercetin prevents glutathione depletion induced by dehydroascorbic acid in rabbit red blood cells," Free Radical Research, vol. 34, no. 6, pp. 639-648, 2001.
[22] M. S. Kim, J. I. Lee, W. Y. Lee, and S. E. Kim, "Neuroprotective effect of Ginkgo biloba L. extract in a rat model of parkinson's disease," Phytotherapy Research, vol. 18, no. 8, pp. 663-666, 2004.

[23] F. Pu, K. Mishima, K. Irie et al., "Neuroprotective effects of quercetin and rutin on spatial memory impairment in an 8arm radial maze task and neuronal death induced by repeated cerebral ischemia in rats," Journal of Pharmacological Sciences, vol. 104, no. 4, pp. 329-334, 2007.

[24] M. M. Ferro, M. I. Bellissimo, J. A. Anselmo-Franci, M. E. M. Angellucci, N. S. Canteras, and C. Da Cunha, "Comparison of bilaterally 6-OHDA- and MPTP-lesioned rats as models of the early phase of Parkinson's disease: histological, neurochemical, motor and memory alterations," Journal of Neuroscience Methods, vol. 148, no. 1, pp. 78-87, 2005.

[25] R. G. M. Morris, "Spatial localization does not require the presence of local cues," Learning and Motivation, vol. 12, no. 2, pp. 239-260, 1981.

[26] H. Ohkawa, N. Ohishi, and K. Yagi, "Assay for lipid peroxides in animal tissues by thiobarbituric acid reaction," Analytical Biochemistry, vol. 95, no. 2, pp. 351-358, 1979.

[27] G. L. Ellman, K. D. Courtney, V. Andres Jr., and R. M. Featherstone, "A new and rapid colorimetric determination of acetylcholinesterase activity," Biochemical Pharmacology, vol. 7, no. 2, pp. 88-95, 1961.

[28] J. M. McCord and I. Fridovich, "Superoxide dismutase. An enzymic function for erythrocuprein (hemocuprein)," Journal of Biological Chemistry, vol. 244, no. 22, pp. 6049-6055, 1969.

[29] A. Wendel, "Glutathione peroxidase," in Enzymatic Basis of Detoxification, W. B. Jakoby, Ed., Academic Press, New York, NY, USA, 1980.

[30] S. A. Goldblith and B. E. Proctor, "Photometric determination of catalase activity," The Journal of biological chemistry, vol. 187, no. 2, pp. 705-709, 1950.

[31] G. Cohen, R. E. Heikkila, and B. Allis, "Destruction of sympathetic nerve terminals by 6 hydroxydopamine: Protection by 1 phenyl 3 (2 thiazolyl) 2 thiourea, diethyldithiocarbamate, methimazole, cysteamine, ethanol and n butanol," Journal of Pharmacology and Experimental Therapeutics, vol. 199, no. 2, pp. 336-352, 1976.

[32] R. Kumar, A. K. Agarwal, and P. K. Seth, "Free radicalgenerated neurotoxicity of 6-hydroxydopamine," Journal of Neurochemistry, vol. 64, no. 4, pp. 1703-1707, 1995.

[33] R. Soto-Otero, E. Méndez-Álvarez, Á. Hermida-Ameijeiras, A. M. Muñoz-Patiño, and J. L. Labandeira-Garcia, "Autoxidation and neurotoxicity of 6-hydroxydopamine in the presence of some antioxidants: potential implication in relation to the pathogenesis of Parkinson's disease," Journal of Neurochemistry, vol. 74, no. 4, pp. 1605-1612, 2000.

[34] Y. Y. Glinka and M. B. H. Youdim, "Inhibition of mitochondrial complexes I and IV by 6-hydroxydopamine," European Journal of Pharmacology, vol. 292, no. 3, pp. 329-332, 1995.

[35] A. Mura and J. Feldon, "Spatial learning in rats is impaired after degeneration of the nigrostriatal dopaminergic system," Movement Disorders, vol. 18, no. 8, pp. 860-871, 2003.

[36] V. Hefco, K. Yamada, A. Hefco, L. Hritcu, A. Tiron, and T. Nabeshima, "Role of the mesotelencephalic dopamine system in learning and memory processes in the rat," European Journal of Pharmacology, vol. 475, no. 1-3, pp. 55-60, 2003.

[37] I. Hernádi, Z. Karádi, J. Vígh et al., "Alterations of conditioned taste aversion after microiontophoretically applied neurotoxins in the medial prefrontal cortex of the rat," Brain Research Bulletin, vol. 53, no. 6, pp. 751-758, 2000. 
[38] T. Florio, A. Capozzo, A. Nisini, A. Lupi, and E. Scarnati, "Dopamine denervation of specific striatal subregions differentially affects preparation and execution of a delayed response task in the rat," Behavioural Brain Research, vol. 104, no. 1-2, pp. 51-62, 1999.

[39] E. De Leonibus, T. Pascucci, S. Lopez, A. Oliverio, M. Amalric, and A. Mele, "Spatial deficits in a mouse model of Parkinson disease," Psychopharmacology, vol. 194, no. 4, pp. 517-525, 2007.

[40] K. Fukui, N. O. Omoi, T. Hayasaka et al., "Cognitive impairment of rats caused by oxidative stress and aging, and its prevention by vitamin E," Annals of the New York Academy of Sciences, vol. 959, pp. 275-284, 2002.

[41] M. Good, "Spatial memory and hippocampal function: where are we now?" Psicologica, vol. 23, no. 1, pp. 109-138, 2002.

[42] M. M. Winnicka and K. Wisniewski, "Bilateral 6-OHDA lesions to the hippocampus attenuate the facilitatory effect of CCK-8US and caerulein on memory in rats," Pharmacological Research, vol. 41, no. 3, pp. 347-353, 2000.

[43] R. D'Hooge and P. P. De Deyn, "Applications of the Morris water maze in the study of learning and memory," Brain Research Reviews, vol. 36, no. 1, pp. 60-90, 2001.

[44] E. K. Perry, M. Curtis, and D. J. Dick, "Cholinergic correlates of cognitive impairment in Parkinson's disease: comparisons with Alzheimer's disease," Journal of Neurology Neurosurgery and Psychiatry, vol. 48, no. 5, pp. 413-421, 1985.

[45] D. A. Drachman and J. Leavitt, "Human memory and the cholinergic system. A relationship to aging?" Archives of Neurology, vol. 30, no. 2, pp. 113-121, 1974.

[46] A. D. Korczyn, "Dementia in Parkinson's disease," Journal of Neurology, Supplement, vol. 248, no. 3, pp. 1-4, 2001.

[47] D. T. Dexter, C. J. Carter, F. R. Wells et al., "Basal lipid peroxidation in substantia nigra is increased in Parkinson's disease," Journal of Neurochemistry, vol. 52, no. 2, pp. 381-389, 1989.

[48] D. T. Dexter, A. E. Holley, W. D. Flitter et al., "Increased levels of lipid hydroperoxides in the Parkinsonian Substantia nigra: an HPLC and ESR study," Movement Disorders, vol. 9, no. 1, pp. 92-97, 1994.

[49] S. J. Kish, C. Morito, and O. Hornykiewicz, "Glutathione peroxidase activity in Parkinson's disease brain," Neuroscience Letters, vol. 58, no. 3, pp. 343-346, 1985.

[50] L. W. Swanson, "The projections of the ventral tegmental area and adjacent regions: a combined fluorescent retrograde tracer and immunofluorescence study in the rat," Brain Research Bulletin, vol. 9, no. 1-6, pp. 321-353, 1982.

[51] L. Walaas, "The hippocampu," in Chemical Neuroanatomy, P. C. Emson, Ed., Raven Press, New York, NY, USA, 1983.

[52] R. D. P. Martins, H. D. C. Braga, A. P. da Silva et al., "Synergistic neurotoxicity induced by methylmercury and quercetin in mice," Food and Chemical Toxicology, vol. 47, no. 3, pp. 645-649, 2009.

[53] B. A. Graf, C. Ameho, G. G. Dolnikowski, P. E. Milbury, C. Y. Chen, and J. B. Blumberg, "Rat gastrointestinal tissues metabolize quercetin," Journal of Nutrition, vol. 136, no. 1, pp. 39-44, 2006.

[54] S. Mandal, B. Hazra, R. Sarkar, S. Biswas, and N. Mandal, "Assessment of the antioxidant and reactive oxygen species scavenging activity of methanolic extract of Caesalpinia crista leaf," Evidence-Based Complementary and Alternative Medicine, vol. 2011, Article ID 173768, 11 pages, 2011.

[55] N. Waksman De Torres, R. Salazar-Aranda, L. A. Pérez-López, J. López-Arroyo, and B. A. Alanís-Garza, "Antimicrobial and antioxidant activities of plants from northeast of Mexico," Evidence-Based Complementary and Alternative Medicine, vol. 2011, Article ID 536139, 6 pages, 2011.

[56] L. Gibellini, M. Pinti, M. Nasi et al., "Quercetin and cancer chemoprevention," Evidence-Based Complementary and Alternative Medicine, vol. 2011, Article ID 591356, 15 pages, 2011.

[57] C. G. M. Heijnen, G. R. M. M. Haenen, F. A. A. Van Acker, W. J. F. Van Der Vijgh, and A. Bast, "Flavonoids as peroxynitrite scavengers: the role of the hydroxyl groups," Toxicology in Vitro, vol. 15, no. 1, pp. 3-6, 2001.

[58] P. S. Naidu, A. Singh, and S. K. Kulkarni, "Reversal of reserpine-induced orofacial dyskinesia and cognitive dysfunction by quercetin," Pharmacology, vol. 70, no. 2, pp. 59-67, 2004.

[59] I. B. Afanas'ev, A. I. Dorozhko, A. V. Brodskii, V. A. Kostyuk, and A. I. Potapovitch, "Chelating and free radical scavenging mechanisms of inhibitory action of rutin and quercetin in lipid peroxidation," Biochemical Pharmacology, vol. 38, no. 11, pp. 1763-1769, 1989.

[60] E. L. Da Silva, M. K. Piskula, N. Yamamoto, J. H. Moon, and J. Terao, "Quercetin metabolites inhibit copper ion-induced lipid peroxidation in rat plasma," FEBS Letters, vol. 430, no. 3, pp. 405-408, 1998.

[61] H. Nagata, S. Takekoshi, T. Takagi, T. Honma, and K. Watanabe, "Antioxidative action of flavonoids, quercetin and catechin, mediated by the activation of glutathione peroxidase," Tokai Journal of Experimental and Clinical Medicine, vol. 24, no. 1, pp. 1-11, 1999.

[62] M. Erden Inal, A. Kahraman, and T. Köken, "Beneficial effects of quercetin on oxidative stress induced by ultraviolet A," Clinical and Experimental Dermatology, vol. 26, no. 6, pp. 536539, 2001.

[63] S. A. Aherne and N. M. O'Brien, "Protection by the flavonoids myricetin, quercetin, and rutin against hydrogen peroxideinduced DNA damage in Caco-2 and Hep G2 cells," Nutrition and Cancer, vol. 34, no. 2, pp. 160-166, 1999.

[64] J. Sanderson, W. R. McLauchlan, and G. Williamson, "Quercetin inhibits hydrogen peroxide-induced oxidation of the rat lens," Free Radical Biology and Medicine, vol. 26, no. 5-6, pp. 639-645, 1999.

[65] E. J. Choi, K. M. Chee, and B. H. Lee, "Anti- and prooxidant effects of chronic quercetin administration in rats," European Journal of Pharmacology, vol. 482, no. 1-3, pp. 281-285, 2003. 


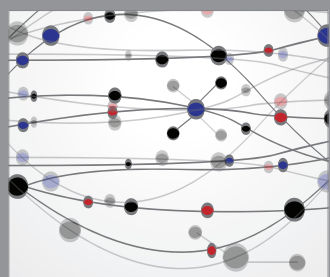

The Scientific World Journal
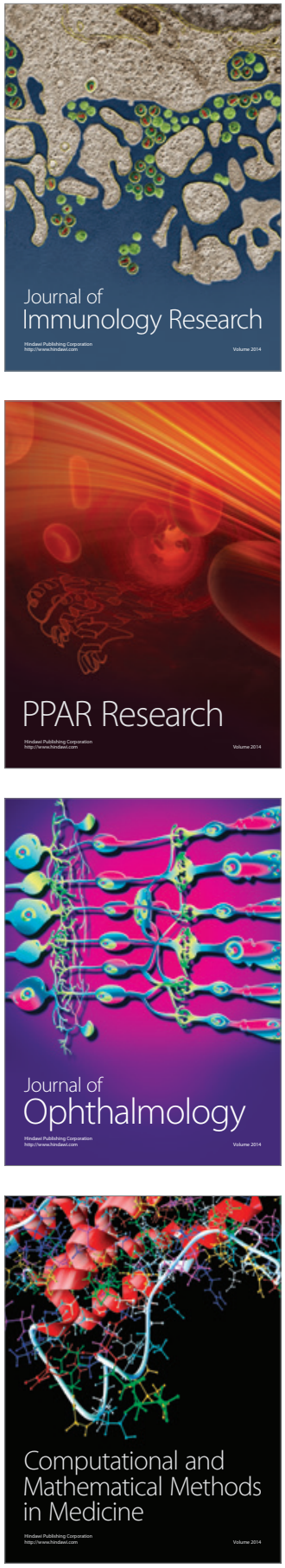

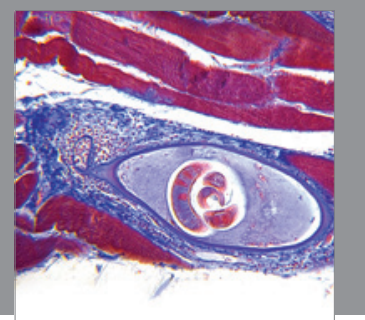

Gastroenterology

Research and Practice
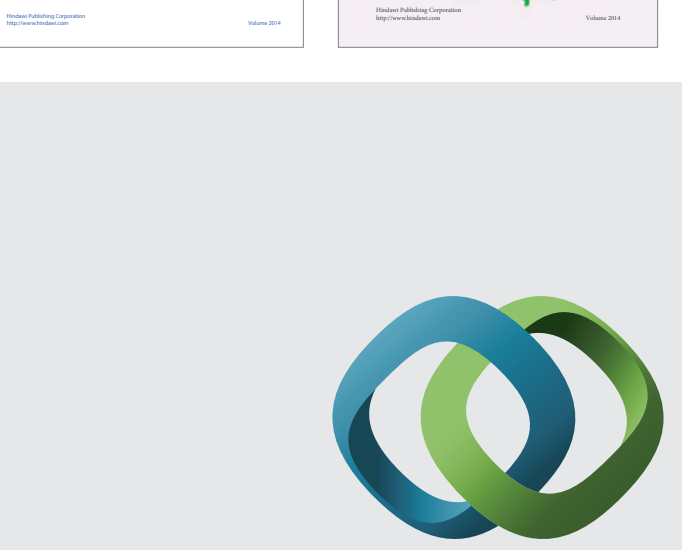

\section{Hindawi}

Submit your manuscripts at

http://www.hindawi.com
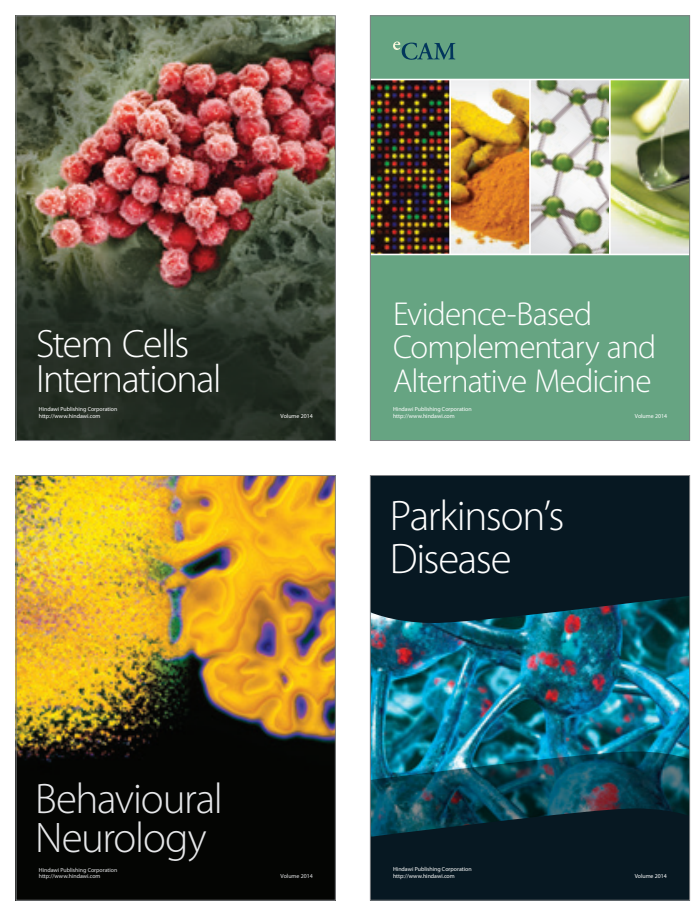

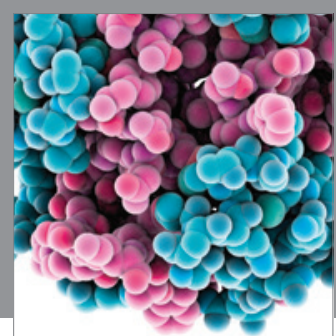

Journal of
Diabetes Research

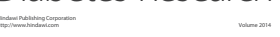

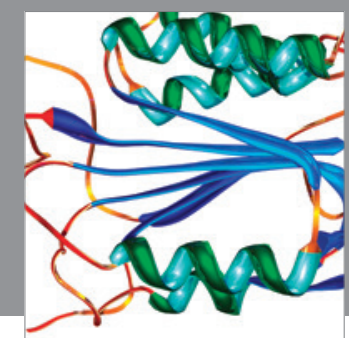

Disease Markers
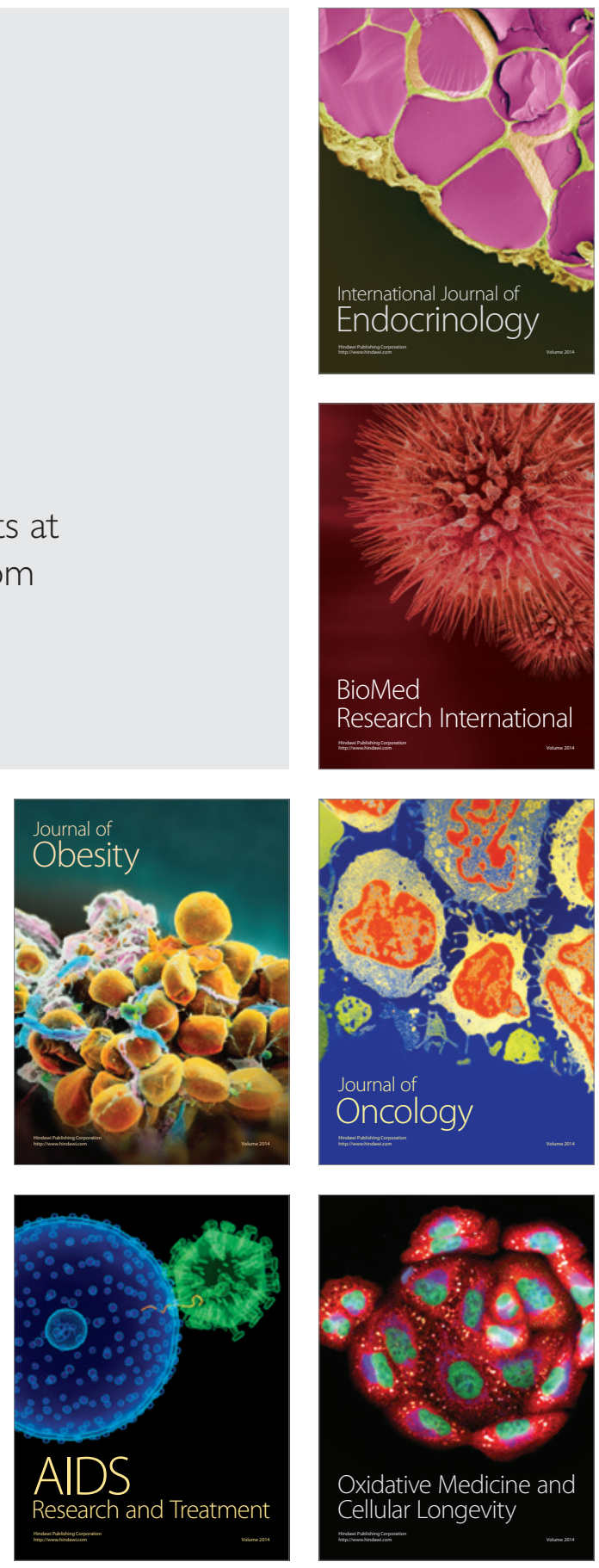